\title{
Decontamination of Chordal Rings and Tori
}

\author{
Paola Flocchini ${ }^{1}$, Miao Jun Huang ${ }^{1}$, Flaminia L. Luccio ${ }^{2}$ \\ ${ }^{1}$ University of Ottawa, \\ ${ }^{2}$ University of Trieste, \\ School of Information Technology and Engineering, Dip. di Matematica e Informatica, \\ Ottawa, Ontario, K1N 6N5, Canada \\ \{flocchin,mhuang\}@site.uottawa.ca \\ 34100, Trieste, Italy \\ luccio@dsm.univ.trieste.it
}

\begin{abstract}
In this paper we consider the problem of decontaminating a network, i.e., protecting it from unwanted and dangerous intrusions. Initially all nodes are contaminated and a team of agents is deployed to clean the entire network. When an agent transits on a node, it can clean it, when the node is left unguarded, however, it will be recontaminated as soon as at least one of its neighbour is contaminated. We study the problem in asynchronous chordal ring networks with $n$ nodes and chord lengths $d_{1}=1, d_{2}, \ldots, d_{k}$, and in tori.

We consider two variations of the model: one where an agent has only local knowledge, the other in which it has "visibility", i.e., it can "see" the state of its neighbouring nodes.

We first show that, when the largest chord $d_{k}$ is not too large $\left(d_{k} \leq \sqrt{n}\right)$, the number of agents necessary to perform the task in chordal rings does not depend on the size of the network but only on the length of the longest chord. We also show a lower bound on the number of agents for the torus topology. We then propose tight strategies for decontamination. We analyse the number of moves and the time complexity of the decontamination algorithms showing that the visibility assumption allows us to decrease substantially both complexity measures. Another advantage of the "visibility model" is that agents move independently and autonomously without requiring any coordination.
\end{abstract}

\section{Introduction}

Problem and Framework. In networked environments supporting mobile agents, security is a pressing

Work partially supported by NSERC, by Dr. Flocchini's University Research Chair, and by AIDA, MIUR COFIN Project. concern and to tackle security issues is becoming more and more complex due to the growing potential threats a network can be faced with. A particularly important security concern is to protect a network from unwanted, and possibly dangerous intrusions. At an abstract level, an intruder is an alien process that moves on the network to sites unoccupied by the system's agents "contaminating" the nodes it passes by. The concern for the severe damage intruders can cause has motivated a large amount of research, especially on detection (e.g., see $[1,11,21])$. Rather than being interested in the detection of the presence of an intruder, we are interested instead in designing strategies for "decontaminating" a possibly infected network, by deploying a team of "cleaning agents". In fact, we consider a networked environment where nodes are hosts and links represent connections between hosts. We assume the nodes of the network are initially contaminated and we want to deploy a team of agents to clean (or decontaminate) the whole network. The cleaning of a node occurs when an agent transit on the node; however, as soon as a node without an agent on it has a contaminated neighbour, it will become contaminated again. We are interested in monotone decontamination strategies, i.e., we want that once a node is clean, it remains clean until the whole network is decontaminated.

More precisely, a team of agents is initially located at a node (the homebase) and agents can move from node to neighbouring node. At any point in time each node of the network can be in one of three possible states: clean, contaminated, guarded. Initially all nodes are contaminated except for the homebase (which is guarded). A node is guarded when it contains at least one agent. We say that a node is clean when an agent passes by it and all its neighbouring nodes are clean or guarded, contaminated otherwise. The solution of the problem is given by devising a strategy for the agents to move in the network in such a way that at the end 
all the nodes are clean.

The system is asynchronous, that is, every action the agents perform (i.e., computing, moving), takes a finite but otherwise unpredictable amount of time. In this setting efficiency is measured in terms of the number of agents to be involved, traffic (i.e., number of moves the agents have to perform), and time (or steps). We consider two variations of the model and accordingly propose lower bounds on the number of agents necessary for decontamination and tight strategies. In the first model (we call it Local model), the only knowledge that an agent has is the information available at its current location (port labels, state of the node); in the second model (Visibility model) agents can "see" also the state of their neighbouring nodes.

Network decontamination could be fairly simple in some specific topologies, where the determination of the minimum number of agents required for the task could be easy. This is the case, for example, of the ring, where two agents starting from the same node can move in opposite direction and easily clean the whole network (with one agent the task would be obviously impossible). Determining the optimal number of agents and a tight strategy is however in general an NP-complete problem.

In this paper we consider mostly chordal ring networks. Chordal rings are a particular case of circulant graphs, and are also known in the literature as distributed loop networks. A chordal ring is a ring augmented by additional chords (each node has the same chord structure) that act as "shortcuts" of the external ring. Chordal rings constitute a common topology for interconnection networks and have been widely studied in the literature to analyse their fault-tolerant properties (for a survey see [5]). Subclasses of chordal rings have been studied under a variety of scenarios and for a large number of problems ranging from routing (e.g., [13])), election (e.g., [2])), broadcast (e.g., [15])).

Related Works. It is easy to see that the decontamination problem can be equivalently formulated in terms of an intruder capture problem, where an intruder moves arbitrarily fast in a network and a team of searching agents is deployed to capture it. The intruder capturing problem has been extensively studied in the literature under the name of graph search in a model where the searchers may be placed and removed from any node of the graph, i.e., they are allowed to "jump" while they perform the searching task. This problem was first introduced by Breish [6]

As the system is asynchronous, we will measure ideal time, i.e., assuming - for the purpose of time complexity only - that it takes one unit of time for an agent to traverse a link. and Parson [18, 19], and after that several variations of the problem have been studied: among them, node search and edge search (see, e.g., [7, 12, 14, 16, 17, 20]), where the aim is to find a strategy that minimizes the number of searchers and leads the graph to a state in which all nodes (or nodes and edges) are simultaneously decontaminated. The size of the searching team is called node-search number $n s(G)$ ( or edge-search number es $(G))$ ) and the determination of the optimal size is an $N P$-complete problem in general.

Graph search, intruder detection, and decontamination are equivalent problems. The main difference in our setting is that the agents cannot be removed from the network: they can only move from a node to a neighbouring node; this assumption is obviously motivated by the fact that we are considering software agents that are able to move only on the edges of the network. In fact, we consider the contiguous, monotone, decontamination first introduced in [3] where: 1) the removal of agents is not allowed, 2) at any time of the search strategy, the set of clean nodes forms a connected subnetwork, and 3) a clean node cannot be recontaminated. The contiguous assumption considerably changes the nature of the problem and the classical results on node and edge search do not generally apply. Moreover, the problem is harder than the noncontiguous one as in [4] it has been proved that the contiguous searching number is always greater or equal to the non-contiguous searching number, the relationship between the search numbers in the two models has been also studied in outerplanar graphs [10]. Finding the contiguous searching number is still an $N P$-complete problem for general graphs; some specific topologies have been studied, for example it has been shown that it can be solved in linear time in trees [3], moreover, optimal strategies have been studied in hypercubes and meshes $[8,9]$, some heuristic algorithms have been discussed in sensor networks [22].

Our Results. As mentioned above, for some topologies an efficient decontamination is easy to perform and finding the optimal number of agents is a trivial task. In general however, the problem is NP-complete. The ring is an extreme case, where decontamination is trivial. Adding extra chords to the ring highly complicates the decontamination problem and clearly only two agents are not sufficient anymore to clean the network. One interesting question that we address in this paper is whether, with the addition of the chords, the minimum number of agents is still constant, or it depends on the size of the network, or on the structure of the chords. Interestingly we show that, when the longest chord is not too long, none of these hypothesis 
is correct; in fact, the smallest number of agents needed for the decontamination solely depends on the length of the longest chord. After we derive the lower bound on the number of agents we describe and analyse two optimal strategies for two variations of the model.

Let $C\left(\left\langle d_{1}=1, d_{2}, \ldots, d_{k}\right\rangle\right)$ be a chordal ring network with $n$ nodes and link structure $\left\langle d_{1}=1, d_{2}, \ldots, d_{k}\right\rangle$, where $d_{i}<d_{i+1}$ and $d_{k} \leq\left\lfloor\frac{n}{2}\right\rfloor$. We first show that, when $4 \leq d_{k} \leq \sqrt{n}$, the minimum number of agents required is $2 \cdot d_{k}$ in the Local model, and it is $2 \cdot d_{k}+1$ in the Visibility model. As a corollary of the lower bounds, we also derive a lower bound for the torus topology (which was unknown) and a simple optimal strategy. We then describe two decontamination algorithms for the chordal ring for the two models, which are optimal in terms of the number of agents.

One of our goals is to understand the power of visibility by determining whether such an assumption can indeed improve the performances of solutions to the problem, and how. In this respect, we have observed that with our strategies the visibility assumption allows to drastically decrease both the time and the number of moves (provided that the longest chord does not exceed $\sqrt{n})$.

\section{Lower Bounds for Decontamination}

Chordal Rings. A circulant graph with $n$ nodes and link structure $\left\langle d_{1}, d_{2}, \ldots, d_{k}\right\rangle, d_{i}<d_{i+1}$, and $d_{k} \leq$ $\left\lfloor\frac{n}{2}\right\rfloor$, is a graph on $n$ nodes $x_{0}, x_{1}, \ldots, x_{n-1}$ where each node $x_{i}$ is adjacent to all the nodes $x_{\left(i+d_{j}\right)} \bmod n$ and $x_{\left(i-d_{j}\right) \bmod n}$ for $1 \leq j \leq k$. A chordal ring is any circulant graph with $d_{1}=1$, i.e., it is an augmented ring and will be denoted by $C\left(\left\langle d_{1}=1, d_{2}, \ldots, d_{k}\right\rangle\right)$. The links of the chordal ring are labeled with chordal sense of direction, i.e., associated to link $\left(x_{i}, x_{j}\right)$ at $x_{i}$ is the distance $(j-i) \bmod n$ between $x_{i}$ and $x_{j}$ along the ring connection.

As the topology is fully symmetric, we can assume that the agents start from any node: the homebase. Let $x_{0}, \ldots x_{n-1}$ be the nodes of the external ring and w.l.g, let $x_{0}$ be the homebase.

In the following we consider the chordal ring as arranged in rows of size $d_{k}$ where the last node of a row is connected to the first node of the following row and the last node is connected to the first. Depending on the size of the chordal ring, the last row could be incomplete. Observe that in this "matrix", going down a column corresponds to using the longest chord $d_{k}$.

The following lower bound holds in chordal rings where, in the arrangement described above, the number of columns is not smaller than the number of rows; in other words, we assume that $d_{k} \leq \frac{n}{d_{k}}$.
Theorem 1. In the Visibility model, any solution of the contiguous decontamination chordal ring $C\left(\left\langle d_{1}=1, d_{2}, \ldots, d_{k}\right\rangle\right)$ with $n$ nodes and $4 \leq d_{k} \leq \sqrt{n}$, requires at least $2 \cdot d_{k}$ searchers.

Proof Let $f$ be the number of clean (or guarded) nodes. Let us consider a subgraph $P\left(\left\langle 1, d_{k}\right\rangle\right)=\left(V, E^{\prime}\right)$ of the chordal ring $C=(V, E)\left(E^{\prime} \subseteq E\right)$ containing only the chords at distance 1 and $d_{k}$. Let us observe the placement of the $f$ clean nodes in $P$. By definition, we know that at any point in time the clean nodes must be connected in $C$; however, in $P$ they might form disconnected blocks. Clearly, the "perimeter" (i.e., the clean nodes of the blocks in contact with contaminated nodes) of these blocks in $P$ must be guarded to avoid recontamination from the neighbouring contaminated nodes (through chords 1 and $d_{k}$ ). First notice that, following a simple geometric reasoning, the number of agents $X$ needed to cover the perimeters of the clean blocks is greater than or equal to the number that it would be required if these blocks were to be attached (i.e., forming a single block) (see Figure 1). Second, it is easy to show that the perimeter of a single block is minimized when it is as close as possible to a rhombus as shown in Figure 2. Let us now compute the number of agents $X$ needed to cover the perimeter of the rhombus; for any other possible shape, $X$ will be a larger number.

Given a rhombus of side $l$, the perimeter is $4 l-4$ and the area (composed of cleaned or guarded nodes) is $f=l^{2}+(l-1)^{2}$. Thus, the number of agents $X$ needed to cover the perimeter of any shape is at least $4 l-4$. From $f=l^{2}+(l-1)^{2}$ we derive that $l=\frac{1+\sqrt{2 f-1}}{2}$; substituting $l$ we have $X \geq 2 \sqrt{2 f-1}-2$.

Consider now a moment during the cleaning when there are $f=l^{2}+(l-1)^{2} \geq \frac{d_{k}^{2}+2 d_{k}+2}{2}$ clean nodes. This holds as long as $n \geq d_{k}^{2} \geq \frac{d_{k}^{2}+2 d_{k}+2}{2}$, i.e., $d_{k} \geq 4$.
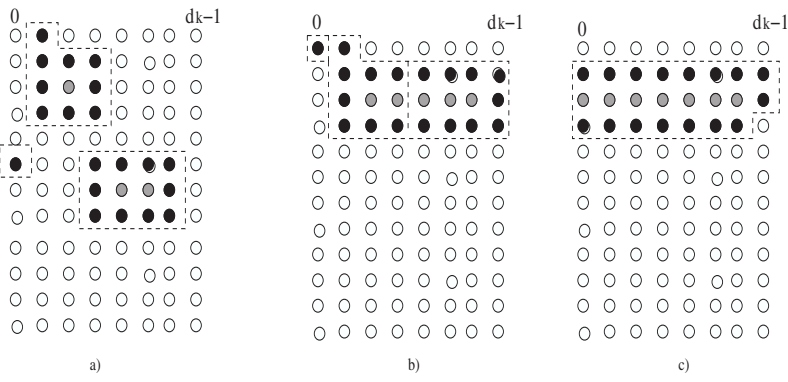

Figure 1. Black nodes are guarded, white nodes are contaminated, grey nodes are clean. 


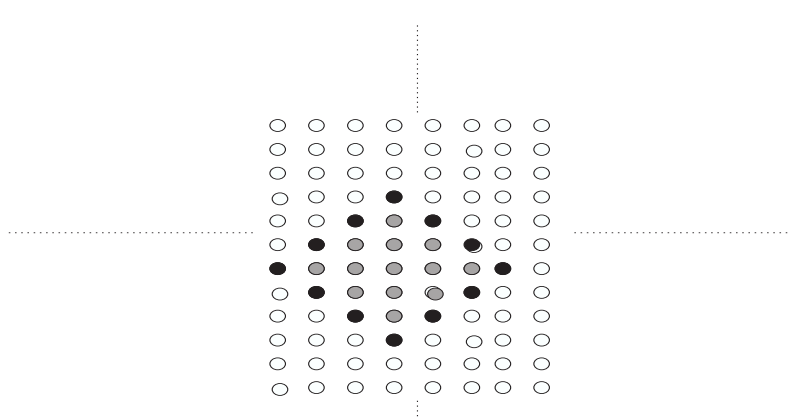

Figure 2. Block that, for a given area, has minimum perimeter.

From $f \geq \frac{d_{k}^{2}+2 d_{k}+2}{2}$, it follows that the number of required agents is $X \geq 2 \sqrt{2\left(\frac{d_{k}^{2}+2 d_{k}+2}{2}\right)-1}-2=2 d_{k}$.

We now show that, when the agents do not have visibility, an additional agent is required to perform the decontamination.

Theorem 2. In the Local model, any solution of the contiguous decontamination problem in a chordal ring $C\left(\left\langle d_{1}=1, d_{2}, \ldots, d_{k}\right\rangle\right)$ with $n$ nodes and $4 \leq d_{k} \leq \sqrt{n}$ requires at least $2 \cdot d_{k}+1$ searchers.

Proof In Theorem 1 we have shown that, at some point, the "perimeter" of the clean area is $2 d_{k}$. Let us assume that the decontamination has reached such a point; i.e., there are $2 \cdot d_{k}$ agents covering the perimeter and let as assume that this number of agents suffices for the decontamination. Since $n$ is big enough compared to $2 d_{k}\left(n \geq \sqrt{d}_{k}\right)$, it is easy to see that, regardless of the shape of the clean area, at least one of the agents (say agent $a$ ) has more than one contaminated neighbour. At least an agent has to move to continue the cleaning. Since the agents have only local knowledge and cannot communicate with each other, let an adversary choose agent $a$ for the next movement. We have a contradiction because the clean area will now be contaminated.

Tori. Following the same lines as the arguments of Theorems 1 and 2, we obtain a lower bound also for the torus, which was not known. The torus is a very common interconnection network; it is the product of two rings and it can be seen as a grid where the last node of a row is connected to the first of the same row, and last node of a column is connected to the first of the same column. Let $T(m, n)$ denote a torus with $m$ rows and $n$ columns, we have that:

Theorem 3. The solution of the contiguous decontamination problem in a torus $T(m, n)$, with $m, n \geq 4$, requires $2 \cdot \min \{m, n\}$ searchers in the Visibility model, and $2 \cdot \min \{m, n\}+1$ searchers in the Local model .

Proof The argument follows the same lines as the argument of Theorems 1 and 2. In this case, however, the "matrix" representation corresponds to the actual torus and there are no additional chords. The minimum perimeter - occurring if the shape of the block were to be a rhombus - would analogously be $4 \sqrt{f}-4$ and the area $f=l^{2}+(l-1)^{2}$, thus the number of required agents would be $X \geq 2 \sqrt{2 f-1}-2$.

Assume $z=\min \{m, n\}$ and consider a moment during the cleaning when there are $f=l^{2}+(l-1)^{2} \geq \frac{z^{2}+2 z+2}{2}$ clean nodes. This holds as long as $m \times n \geq z^{2} \geq$ $\frac{z^{2}+2 z+2}{2}$, i.e., $z=\min \{m, n\} \geq 4$.

From $f \geq \frac{z^{2}+2 z+2}{2}$, it follows that the number of required agents is $X \geq 2 \sqrt{2\left(\frac{z^{2}+2 z+2}{2}\right)-1}-2=2 z=$ $2 \min \{m, n\}$, and the number of required agents is at least $2 \min \{m, n\}$.

If there is no visibility (Local model) an additional agent is required following the same reasoning of Theorem 2 .

\section{Optimal Decontamination Strategies}

\subsection{Chordal Rings}

In this section we propose two algorithms respectively for the capture of the intruder in a chordal ring of size $n$ in the Local and in the Visibility models.

In the following discussion, we will assume that operation among indices are modulo $n$ ( i.e., $i+d_{j}$ means $\left.\left(i+d_{j}\right) \bmod n\right)$. We call clockwise neighbors (respectively, counterclockwise neighbors) of $x_{i}$, the set of neighbors $\left\{x_{i+1}, x_{i+d_{2}}, \ldots, x_{i+d_{k}}\right\}$ (respectively, $\left\{x_{i-1}, x_{i-d_{2}}, \ldots, x_{i-d_{k}}\right\}$ ). We call window of size $s$ is a sequence of $s$ nodes in consecutive clockwise positions along the ring.

Decontamination with a Coordinator. The first strategy we present is for the Local model, where a special agent is designated as a coordinator. The main idea is that the searching agents are coordinated by this special agent that, moving back and forth, allows them to visit all nodes and safely protects the system from the recontamination. 
Cleaning strategy. In this strategy we employ $2 d_{k}$ identical agents and a coordinator. We assume that the coordinator can communicate with an agent when they reside on the same node.

The cleaning must be preceded by a deployment stage after which the agents have to occupy $2 d_{k}$ consecutive nodes. The simplest way to deploy the agents is for the coordinator to lead them to their respective starting position by moving along the external ring. During the deployment the agents also clean the $2 d_{k}$ nodes and no recontamination occurs (i.e., the deployment is monotone). After the deployment, nodes $x_{0}$ to $x_{2 d_{k}-1}$ are guarded by one agent, and the coordinator moves to node $x_{d_{k}}$, which is then guarded by two agents. During the cleaning stage, node $x_{0}$ to $x_{d_{k}-1}$ are still guarded by one agent each, forming a window of $d_{k}$ agents. This window of agents shields the clean nodes from recontamination from one direction of the ring while the agents of the other window are moved by the coordinator (one at a time) along their longest chord to clean the next window in the ring.

\section{Algorithm 1 Clean with a Coordinator.}

Initially, nodes $x_{0} \ldots x_{2 d_{k}-1}$ are guarded by an agent each and the coordinator is at $x_{d_{k}}$.

1.1 The cleaning starts at $x_{d_{k}}$ and proceeds in the clockwise direction until $x_{n-1-d_{k}}$ is reached. Let $x_{i}\left(d_{k} \leq i \leq n-1-d_{k}\right)$ be the node with an agent and the coordinator on it. The two agents move along link $d_{k}$ of $x_{i}$, and only when they both arrive at node $x_{i+d_{k}}$, one is left to guard $x_{i+d_{k}}$ and the coordinator first goes back to $x_{i}$ and then moves along link 1 of $x_{i}$ to arrive at node $x_{i+1}$. The agent on any node $x_{j}$ where $i \neq j$ has to wait on $x_{j}$ for the coordinator to arrive.

1.2 When two agents are on node $x_{n-d_{k}}$, one agent terminates and the coordinator goes to notify all the agents on node $x_{n-d_{k}+1}$ to $x_{d_{k}-1}$ to terminate.

Note that in Step 1.2., when two agents are on node $x_{n-d_{k}}$ all nodes from $x_{n-d_{k}}$ to $x_{n}$ are guarded. Note also that as the system is asynchronous, in the different phases the coordinator has to make sure every agent reaches its position before the new step can start.

Correctness and Complexity. We first prove that our cleaning strategy is correct; i.e., that all nodes will be cleaned and that once a node has been cleaned, it will never be recontaminated.
Theorem 4. Algorithm 1 cleans all the nodes of the chordal ring and a clean node will never be recontaminated.

Proof We first prove that a clean node will not be recontaminated. By induction.

The cleaning starts at node $x_{d_{k}}$. Except the neighbor $x_{2 d_{k}}$ of $x_{d_{k}}$, which is not guarded by an agent yet, all the other neighbors are guarded. By the strategy, the two agents on it move to $x_{2 d_{k}}$, the only contaminated neighbor. Then one agent is left to guard this neighbor and the other goes back to $x_{d_{k}}$. So all neighbors of $x_{d_{k}}$ are guarded; when the agent from $x_{2 d_{k}}$ comes back to $x_{d_{k}}$ and then moves to $x_{d_{k}+1}$, node $x_{d_{k}}$ becomes clean and no recontamination can occur.

Assume nodes $x_{d_{k}}$ to $x_{i-1}$ where $d_{k} \leq i-1 \leq n-2-d_{k}$ are clean and the cleaning is at node $x_{i}$. We show that $x_{i}$ becomes clean and no recontamination can occur. By the first step of the strategy and by the induction hypothesis, node $x_{0}$ to $x_{i-1}$ are clean or guarded. So the counterclockwise neighbors of $x_{i}$ are all either guarded or clean. Moreover, we know nodes from $x_{i}$ to $x_{i-1+d_{k}}$ are guarded. So the clockwise neighbors of $x_{i}$ are guarded except $x_{i+d_{k}}$. By the strategy, when the two agents move to the only contaminated neighbor $x_{i+d_{k}}$, no recontamination can occur. One agent is left to guard $x_{i+d_{k}}$. So all neighbors of $x_{i}$ are either guarded or clean; when the agent goes back to $x_{i}$ and then moves to $x_{i+1}$, no agent is left to guard $x_{i}$ and $x_{i}$ becomes clean. No recontamination can occur to $x_{i}$.

At step 1.2, when the agent from $x_{n-1-d_{k}}$ arrives at $x_{n-d_{k}}$, all the nodes from $x_{n-d_{k}}$ to $x_{d_{k}-1}$ are guarded and the others are clean from step 1.1. There is no contaminated node anymore and it is impossible to recontaminate a clean node. Since a clean node will never be recontaminated, by cleaning strategy after step 1.2, all agents terminate and all the nodes become clean.

We now compute the number of moves performed by the agents during the cleaning.

Theorem 5. The total number of moves performed by the agents is $4 n-6 d_{k}-1$.

Proof For cleaning a node $x_{i}$, four moves are performed by the agents. It takes one move for each of the two agents to arrive to $x_{i+d_{k}}$, one move back to $x_{i}$ and then one move to $x_{i+1}$. So totally, $4\left(n-2 d_{k}\right)$ moves are performed in the cleaning stage. It takes $2 d_{k}-1$ moves for the agent from $x_{n-d_{k}}$ to notify agents on $x_{n-d_{k}}$ to $x_{d_{k}-1}$ to terminate. So totally, the number of moves for the entire process is $4\left(n-2 d_{k}\right)+$ $2 d_{k}-1=4 n-6 d_{k}-1$.

We now consider the ideal time complexity of the cleaning strategy. 
Theorem 6. The cleaning strategy takes $3 n-4 d_{k}-1$ time units.

Proof The cleaning process is carried out sequentially by the coordinator agent on each node. The time required is then equal to the number of moves of the coordinator, which is $3\left(n-2 d_{k}\right)+2 d_{k}-1$. So totally, it takes $3\left(n-2 d_{k}\right)+2 d_{k}-1=3 n-4 d_{k}-1$.

Finally, it directly follows from the strategy that:

Theorem 7. Our strategy employs $2 d_{k}+1$ agents.

Decontamination with Visibility. In this section we consider the decontamination problem in the Visibility model: we assume that an agent located at a node can "see" whether its neighboring nodes are clean or guarded or contaminated. This capability could be easily achieved if the agents have communication power and send a message (e.g., a single bit) to their neighbouring nodes after cleaning a node or guarding a node. The interesting aspect of this model is that this extra capability enables agents to correctly act without the need of being coordinated. We also assume that agents have distinct Ids, otherwise they cannot perform any meaningful computation starting from the same homebase (symmetry could not be broken).

Cleaning strategy. The idea of the algorithm is quite simple and all the agents follow the same local rule: as soon as an agent sees that all its neighbours are clean except for one, it moves there. Before starting the algorithm, however, a deployment phase is needed during which the agents move to occupy $2 d_{k}$ consecutive nodes. As for the Local model, we want that they deploy in a monotone way, that is during the deployment they also start cleaning the nodes without allowing any recontamination. For example, we can assume they move along the external ring occupying one node each like in the deploy for the Local model.

\section{Algorithm 2 Clean With VISIBILITY.}

Initially all agents are in $x_{0}$ and they start the deployment to occupy $x_{n-\left(d_{k}-1\right)}, \ldots, x_{d_{k}}$.

- When an agent on a node $x_{i}$ "sees" that node $x_{i}$ has only one contaminated neighbor, the agent moves to clean the contaminated neighbor; when the agent "sees" that all the neighbors are clean or guarded, it terminates; otherwise, it waits on the node.

Notice that the execution of the cleaning algorithm could actually start before the deployment is completed. In fact, as soon as an agent sees it has only one contaminated neighbour, it can start the cleaning.
Correctness and Complexity. We first prove that our strategy is correct, i.e., that the network is clean and once a node has been cleaned, it will never be recontaminated.

Lemma 1. In a chordal ring network with $n$ nodes and link structure $\left\langle d_{1}=1, d_{2}, \ldots, d_{k}\right\rangle, d_{i}<d_{i+1}$, and $d_{k} \leq\left\lfloor\frac{n}{2}\right\rfloor$, within a window of size $2 d_{k}$, there are at most $2\left(d_{k}-d_{k-1}\right)$ nodes which have only one neighbor outside this window. These nodes are consecutive along the external ring.

Proof Let us arbitrarily pick a window $W$ of size $2 d_{k}$ and mark the first node to be $x_{n-\left(d_{k}-1\right)}$ and the last node to be $x_{d_{k}}$. We can also see $W$ as two windows $W_{1}$ and $W_{2}$ of size $d_{k}$ and such that $W=W_{1} \bigcup W_{2}$. Window $W_{1}$ covers nodes from $x_{n-\left(d_{k}-1\right)}$ to $x_{0}$ and $W_{2}$ covers nodes from $x_{1}$ to $x_{d_{k}}$. Inside $W_{1}$, nodes from $x_{n-d_{k}+d_{k-1}+1}$ to $x_{0}$ have only one neighbor outside $W=W_{1} \bigcup W_{2}$ in the counterclock direction. Similarly, inside $W_{2}$, nodes from $x_{1}$ to $x_{d_{k}-d_{k-1}}$ have only one neighbor outside of $W$ in the clockwise direction. So totally, there are at most $2\left(d_{k}-d_{k-1}\right)$ nodes having only one neighbor outside of $W$.

Observe now that if $n<4 d_{k}-2 d_{k-1}$ some of the nodes that had to be outside the window $W$, lie on the opposite subwindow (i.e., from $W_{1}$ to $W_{2}$ and vice versa). Thus, we may obtain the following:

Corollary 1. If $n \geq 4 d_{k}-2 d_{k-1}$, within a window of size $2 d_{k}$ there are exactly $2\left(d_{k}-d_{k-1}\right)$ consecutive nodes which have only one neighbor outside this window.

We now prove the correctness of the strategy.

Theorem 8. Algorithm 2 cleans all the nodes of the chorded ring and a clean node will never be recontaminated.

Proof We have to prove both that the strategy considers all the nodes of the chordal and that no recontamination occurs.

Let us first prove by induction that there is no recontamination, i.e., a clean node may never be a neighbour of a contaminated node. To prove this we also prove something else, i.e., that if an agent has left a node $x_{i}$ reached during the deployment stage to move to a new node for the cleaning stage, then $x_{i}$ is not a neighbour of a contaminated node. This second property is included in the first but considering it apart will help us during the proof.

We start the induction considering the initial state during which all the agent are placed on the homebase. There is only one guarded node and all the other nodes are contaminated, thus the two property hold. Consider the system after a set of moves during which both 
properties held and let us now consider a set of agents that execute a new move. There are two possible cases depending on the fact that an agent that moves is in the deployment or in the cleaning stage. If the agent is in the deployment stage, this holds because the deployment strategy is monotone. So the case we are really interested in is the one in which a node moves during the cleaning stage. Since no recontamination has occurred previously, the move is safe because an agent moves only if all the neighbours but one are clean or guarded and it moves towards the contaminated node. Thus, no recontamination occurs.

Let us now prove that the strategy cleans all the nodes, i.e., all nodes are eventually reached. After the deployment stage all nodes in a window $W$ of size $2 d_{k}$ starting from $x_{n-\left(d_{k}-1\right)}$ and ending in $x_{d_{k}}$ are guarded and thus cleaned by an agent. By lemma 1 we know that there are $2\left(d_{k}-d_{k-1}\right)$ consecutive nodes which have only one neighbor outside window $W$, thus agents guarding these nodes will eventually move to guard $\left(d_{k}-d_{k-1}\right)$ nodes outside $W$ on the left and $\left(d_{k}-d_{k-1}\right)$ nodes on the right forming a clean window $W^{\prime}$ of size $4 d_{k}-2 d_{k-1}$. There are now other $2\left(d_{k}-d_{k-1}\right)$ nodes of $W^{\prime}$ which have only one neighbor outside $W^{\prime}$ (this time the nodes are not consecutive, but they form two consecutive blocks of size $\left(d_{k}-d_{k-1}\right)$, thus this procedure enlarges the window up to when the whole chordal ring is covered.

We now consider the time complexity of the cleaning strategy. The time complexity is the ideal time complexity which is computed by assuming that it takes one unit of time for an agent to traverse an edge. The computation starts at $x_{0}$.

Theorem 9. The cleaning strategy takes at most $\left\lceil\frac{n-2 d_{k}}{2\left(d_{k}-d_{k-1}\right)}\right\rceil$ time units.

Proof For simplicity, let us assume that the cleaning starts after the deployment is completed (as we have seen before some agents could actually start earlier). We also assume that $n>2 d_{k}$, otherwise nothing has to be done after the deployment phase. We can now divide our computation after the deployment phase into different sub-phases during which, using lemma 1 , blocks of $2\left(d_{k}-d_{k-1}\right)$ nodes are cleaned until the whole network is cleaned. Thus, in at most $\left[\frac{n-2 d_{k}}{2\left(d_{k}-d_{k-1}\right)}\right]$ time steps blocks of $2\left(d_{k}-d_{k-1}\right)$ nodes are cleaned (one in each time step) up to when the whole network is cleaned.

Theorem 10. The total number of moves performed by the agents during the cleaning strategy is at most $n-2 d_{k}$.
Proof It directly follows from the strategy that asserts that during the cleaning stage an agent moves to a node only if it "sees" that is it the only contaminated neighbour, thus it only moves to contaminated nodes. Moreover from Theorem 8 we know that the strategy is correct and all the contaminated nodes will be cleaned. Thus all contaminated nodes will be visited exactly once, and after the cleaning phase there are $n-2 d_{k}$ of them (the remaining nodes are guarded).

\subsection{Torus}

In this section we mention very briefly the decontamination strategies that match the lower bounds for the tori. The algorithms are very similar to the ones for the mesh topology described in [9]. Without loss of generality, let us assume that the number of rows is smaller than the number of columns. The idea is to deploy the agents to cover two consecutive columns and then keep one column of agents to guard from decontamination and have the other column move along the torus. In the Local model the movement of the agents must be synchronized by a coordinator so to avoid recontamination, in the Visibility model the agents can safely move autonomously.

The cleaning is identical to the one for the mesh, the only difference is that in the mesh it was not required to have a column of agents shielding one part of the network from recontamination since the cleaning could start from a border of the mesh. The move and time complexities are the same as in [9], and are reported in Table 1 (where we assume, w.l.g., that $m=\min \{m, n\}$ and we consider the complexity of the cleaning only).

\section{Remarks and Open Problems}

In this paper we have considered the problem of decontamination in chordal rings. We have determined a lower bound on the number of agents required and two strategies in two variations of the model. Our results on the cost of the cleaning strategy are summarized in Table 2 .

Following similar reasonings as the ones of the lower bounds for the chordal ring, we have obtained lower

\begin{tabular}{|c|c|c|c|}
\hline Torus & Agents & Time & Moves \\
\hline Coord. & $2 m+1$ & $m n-2 m$ & $2 m n-4 m-1$ \\
\hline Visib. & $2 m$ & $n-2$ & $m n-2 m$ \\
\hline
\end{tabular}

Table 1. Results for the Torus. 


\begin{tabular}{|c|c|c|c|}
\hline Ch. Ring & Agents & Time & Moves \\
\hline Coord. & $2 d_{k}+1$ & $4 n-6 d_{k}-1$ & $3 n-4 d_{k}-1$ \\
\hline Visib. & $2 d_{k}$ & $\frac{n-2 d_{k}}{2\left(d_{k}-d_{k-1}\right)}$ & $n-2 d_{k}$ \\
\hline
\end{tabular}

Table 2. Results for the Chordal Ring.

bounds also for the Torus (which were not known); these bounds can be easily matched by simple cleaning strategies.

We have observed that with our strategies the visibility assumption allows us to drastically decrease the time and move complexities in both topologies. In particular, the strategies for the Visibility model are optimal both in terms of number of agents and in terms of number of moves; as for the time complexity, visibility allows some concurrency. For example, in the case of the chordal ring the level of concurrency depends on the distance between the longest chord and the second longest. The higher the distance, the higher the concurrency is, and thus the improvement in time complexity. The improvements hold when the longest chord $d_{k}$ is such that $4 \leq d_{k} \leq \sqrt{n}$, otherwise our strategies are not efficient and the lower bound is not valid. Consider, for example, the case of $C(\langle 1,8\rangle)$ with 24 nodes. It is easy to see that the decontamination can be done with 6 agents only (placed in two consecutive "columns" in the matrix representation), while our bound would prescribe 16 . The determination of the minimum number of agents needed when $d_{k}>\sqrt{n}$ and a matching strategy is an interesting open problem.

Another interesting problem would be to study a trade-off between time and number of agents. For example, if we add one extra agent to our strategy with coordinator, that is we employ $2 d_{k}+2$ agents, we can reduce the cleaning process time by half. The extra agent can move in opposite direction to clean the contaminated nodes thus two agents would be cleaning concurrently, in the two directions of the external ring.

\section{References}

[1] M.Asaka, S.Okazawa, A.Taguchi, S.Goto. A method of tracing intruders by use of mobile agents, 9th Annual Internet Society Conference (INET), 1999.

[2] H. Attiya, J. van Leewen, N. Santoro, S. Zaks. Efficient elections in chordal ring networks. Algorithmica, 4:437-446, 1989.

[3] L. Barrière, P. Flocchini, P. Fraigniaud, N. Santoro. Capture of an intruder by mobile agents. 14-th $A C M$ Symposium on Parallel Algorithms and Architectures (SPAA), 200-209, 2002.
[4] L. Barrière, P. Fraigniaud, N. Santoro and D.M. Thilikos. Searching is not jumping. 29th Workshop on Graph Theoretic Concepts in Computer Science (WG), LNCS 2880, 34-45, 2003.

[5] J-C Bermond, F. Comellas, D.F. Hsu. Distributed loop computer networks: a survey. Journal of Parallel and Distributed Computing, 24:2-10, 1995.

[6] R. Breish. An intuitive approach to speleotopology. Southwestern cavers, VI (5), 72-28, 1967.

[7] J.A. Ellis, I.H. Sudborough, J.S. Turner. The vertex separation and search number of a graph. Information and Computation, 113: 50-79, 1994.

[8] P. Flocchini, M.J. Huang, F.L. Luccio. Contiguous search in the hypercube for capturing an intruder. 19th IEEE International Parallel and Distributed Processing Symposium (IPDPS), 2005.

[9] P. Flocchini, F.L. Luccio, L.X. Song. Size optimal strategies for capturing an intruder in mesh networks. International Conference on Communications in Computing (CIC), 200-206, 2005.

[10] F.V. Fomin, D.M. Thilikos, I. Todinca. Connected graph searching in outerplanar graphs. 7th Int. Colloquium on Graph Theory (ICGT), Electronic Notes in Discrete Mathematics 22, 213-216, 2005.

[11] N. Foukia, J.G.Hulaas, J. Harms. Intrusion Detection with Mobile Agents. 11th Annual Internet Society Conference (INET), 2001.

[12] L. M. Kirousis,C. H. Papadimitriou. Searching and pebbling. Th. Computer Science, 47:205-218, 1986.

[13] D. Krizanc, F.L. Luccio. Boolean routing on chordal ring. 2nd Int. Colloquium on Structural Information and Communication Complexity, 89-100, 1995.

[14] A. Lapaugh. Recontamination does not help to search a graph. Journal of the ACM, 40(2): 224-245, 1993.

[15] B. Mans. Optimal distributed algorithms in unlabeled tori and chordal rings. Journal of Parallel and Distributed Computing, 46(1): 80-90, 1997.

[16] N. Megiddo, S. Hakimi, M. Garey, D. Johnson, C. Papadimitriou. The complexity of searching a graph. Journal of the ACM, 35(1): 18-44, 1988.

[17] B. Monien, I.H. Sudborough. Min cut is NP-complete for edge weighted trees. Theoretical Computer Science, 58, 209-229, 1988.

[18] T. Parson. Pursuit-evasion problem on a graph. Theory and applications in graphs, Lecture Notes in Mathematics, Springer-Verlag, 426-441, 1976.

[19] T. Parson. The search number of a connected graph. 9th Southeastern Conference on Combinatorics, Graph Theory and Computing, Utilitas Mathematica, 549$554,1978$.

[20] S. Peng, M. Ko, C. Ho. T. Hsu, C. Tang. Graph searching on some subclasses of chordal graphs. Algorithmica, 27: 395-426, 2000.

[21] E.H. Spafford, D. Zamboni. Intrusion detection using autonomous agents, Computer Networks, 34(4), 547570, 2000.

[22] P. Yospanya, B. Laekhanukit, D. Nanongkai, J. Fakcharoenphol. Detecting and cleaning intruders in sensor networks. 8th National Computer Science and Engineering Conference, 2004. 